\title{
Metodologias Ativas no Ensino Superior: Percepção Docente sobre a Importância da Continuidade do Processo de Aprendizagem
}

\author{
Rodolfo de Oliveira Medeiros ${ }^{1}$, Elza de Fátima Ribeiro Higa', Maria \\ José Sanches Marin ${ }^{1}$, Carlos Alberto Lazarini ${ }^{1}$ e Monike Alves \\ Lemes $^{1}$
}

\author{
1 Faculdade de Medicina de Marília, Brasil | rodorfo.famema@hotmail.com; \\ hirifael@gmail.com; marnadia@terra.com.br; carlos.lazarini@gmail.com; \\ monikealvesx3@gmail.com | https://orcid.org/0000-0002-4930-684X; https://orcid.org/0000- \\ 0001-5772-9597; https://orcid.org/0000-0001-6210-6941; https://orcid.org/0000-0003-3010- \\ 4436; https://orcid.org/0000-0002-8769-0993
}

\begin{abstract}
Resumo: Introdução: A educação pode ser considerada um caminho para mudar o mundo. Para isso, busca proporcionar, de forma eficaz, cada vez mais conhecimentos que são as bases para o desenvolvimento integral humano. Neste tocante, a formação continuada de docentes figura como importante aspecto na busca por mudanças significativas no cenário educacional, considerando as especificidades de cada cenário de sua atuação. As metodologias ativas no ensino superior têm despontado como uma estratégia inovadora e ao mesmo tempo desafiadora. Para tanto, faz-se necessário que o docente inserido nesse cenário esteja em constante aprendizado. Objetivo: Compreender a visão dos docentes do ensino superior em saúde sobre a continuidade do processo de aprendizagem. Método: Pesquisa qualitativa fundamentada nos pressupostos da Teoria das Representaç̃es Sociais, realizada em uma Faculdade do interior paulista, com quarenta docentes dos cursos de medicina e enfermagem, inseridos em unidades educacionais distintas. A coleta de dados ocorreu a partir de entrevistas gravadas, com uma questão norteadora que buscou compreender a percepção docente acerca da continuidade do processo de aprendizagem no âmbito das metodologias ativas. Resultados: Segundo a percepção dos docentes entrevistados, a continuidade do processo de aprendizagem é fundamental, essencial e oportuna, proporcionando condições de estarem sempre atualizados frente às inovações da atualidade. Indicaram as Tecnologias Digitais de Informação e Comunicação a principal forma de continuar aprendendo Conclusões: Nota-se que a percepção docente acerca da continuidade do processo de aprendizagem consiste em mecanismos de atualização. Reiterase a necessidade de as instituições de ensino superior ofertarem condições para que ocorra a continuidade do processo de atividade docente.
\end{abstract}

Palavras-chave: Saúde. Formação de Professores. Educação Permanente.

Active Methodologies in Higher Education: Teacher's Perception on the Importance of the Continuity of the Learning Process

\begin{abstract}
Introduction: Education can be considered a way to change the world. To this end, it seeks to provide, in an effective manner, more and more knowledge that is the basis for integral human development. In this regard, the continuing education of teachers appears as an important aspect in the search for significant changes in the educational scenario, considering the specificities of each scenario of their performance. Active methodologies in higher education have emerged as an innovative and challenging strategy. Therefore, it is necessary that the teacher inserted in this scenario is in constant learning. Objective: To understand the view of higher education teachers in health on the continuity of the learning process. Method: Qualitative research based on the assumptions of the Theory of Social Representations, carried out at a Faculty in the interior of São Paulo, with forty professors from the medical and nursing courses, inserted in different educational units. Data collection took place from recorded interviews, with a guiding question that sought to understand the teaching perception about the continuity of the learning process within the scope of active methodologies. Results: According to the perception of the professors interviewed, the continuity of the learning process is fundamental, essential and timely, providing conditions to be always up-to-date in the face of current innovations. Digital Information and Communication Technologies indicated the main way to continue learning. Conclusions: It is noted that the teaching perception about the continuity of the learning process consists of updating mechanisms. The need for higher education institutions to offer conditions for the continuity of the teaching activity process is reiterated.
\end{abstract}

Keywords: Health. Teacher training. Permanent Education. 


\section{Introdução}

A educação consiste num processo contínuo, que ocorre do começo ao fim da vida, no qual o indivíduo se atualiza a medida em que se relaciona com o meio em que o cerca. A premissa de aprendizagem ao longo da vida foi preconizada pela Organização das Nações Unidas para a Educação, a Ciência e a Cultura (Unesco), apontando para a educação humana organizada a partir de quatro pilares: Aprender a conhecer, aprender a fazer, aprender a viver juntos e a aprender a ser (DELORS, 2003).

De forma ampla, o ensino formal está voltado, essencialmente, para o aprender a conhecer e, em menor escala, aprender a fazer. Porém, é necessária a compreensão de que os quatro pilares do conhecimento devem ser considerados de igual forma por parte do ensino estruturado, objetivando a ótica da educação como uma experiência global ao longo de toda a vida, seja nos domínios cognitivos ou práticos, considerando o ser humano enquanto pessoa e membro de uma sociedade (DELORS, 2003).

No contexto da história da educação, ao se compreender o processo no qual se desenvolveram as tendências pedagógicas ao longo da história, sendo a maioria pautada em um ensino técnico, tradicional e de memorização e conteúdo, justifica-se a ideia de mudança no processo de construção de conhecimento, surgem os Métodos de Aprendizagem Ativa (PIO, 2017).

A metodologia proporciona protagonismo ao estudante, a partir do estímulo ofertado pelo tutor mediador no processo de ensino e aprendizagem, onde é possível os discentes realizarem apropriação do conhecimento a partir de suas intencionalidades (MACEDO et al., 2018; HIGA et al., 2018). A estrutura curricular subsidia-se na interdisciplinaridade, ou seja, na integração de conteúdo das diversas áreas do saber, orientado por competência profissional e centrado no estudante (TAROCO, TSUJI, HIGA, 2017; TEÓFILO, SANTOS, BADUY, 2017).

Nesses espaços, o estudante utiliza seus conhecimentos prévios para interpretar uma situação apresentada, normalmente em forma de problema, objetivando buscar meios para solucioná-lo (PINTO, MISTRO, UEMURA, 2016), sendo o docente, segundo a teoria piagetiana, facilitador do processo de ensino e aprendizagem, com o intuito de estimular e instigar o discente na busca pelo saber, contribuindo para a formação de sujeitos críticos e reflexivos (LARA et al., 2019; HIGA et al., 2016).

No campo das metodologias ativas, diversas estratégias de aprendizagem têm norteado o ensino universitário em vários países, e dentre elas apontam: Aprendizagem Baseada em Problemas (APB), Metodologia da Problematização, Team-Based Learning e Sala de Aula Invertida (GODINHO et al., 2017; OLIVEIRA, LIMA, RODRIGUES, JUNIOR, 2018).

Neste campo do saber, a formação docente deve ocorrer de maneira contínua, não por acúmulo de cursos, mas por meio de processos reflexivos acerca das práticas, que se inicia com a formação inicial e acompanha o processor durante toda a sua trajetória (ARAÚJO et al., 2016; FALSARELLA, 2004). Em tempos de mudanças rápidas e contínuas no cenário educacional, é importante o docente manter-se em permanente formação, através de processos que envolvem pesquisa, ação, descoberta, fundamentação e construção teórica, para que o mesmo se mantenha atualizado ao longo de sua trajetória (FREITAS, PACÍFICO, 2020). Diante disso, é evidente a importância da continuidade do processo de aprendizagem de docentes inseridos em métodos de aprendizagem ativa, para que os mesmos possam contribuir para o processo de ensino e aprendizagem (TSUJI, AGUILAR-DA-SILVA, 2010).

Frente ao exposto, observam-se questões que dizem respeito a formação continuada em serviço e a necessidade da continuidade do processo de aprendizagem docente. No entanto, a busca pela reflexão sobre a questão posta emergiu da seguinte inquietude: qual a compreensão de docentes do ensino superior em saúde sobre continuidade do processo de aprendizagem? 


\section{Objetivo}

Compreender a visão dos docentes do ensino superior em saúde sobre a continuidade do processo de aprendizagem.

\section{Método}

Pesquisa qualitativa, fundamentada nos pressupostos da Teoria das Representações Sociais (TRS). Segundo Minayo (2013), a pesquisa qualitativa consiste em um método que se apropria do nível subjetivo e relacional da realidade social, estudando fenômenos e relações em seu meio natural, valorizando o universo dos significados, dos motivos, das crenças, dos valores e das atitudes dos atores sociais, com potencial para desvendar processos sociais pouco conhecidos no contexto de grupos sociais específicos.

Desenvolvida a partir da sociologia clássica de Durkhein e resgatada anos mais tarde por Serge Moscovici, a Teoria das Representações Sociais, conotada como um fenômeno social, possui como questão norteadora a compreensão do conhecimento da vida real cotidiana, a partir de teorias construídas pelo senso comum para ilustrar as realidades de determinados grupos sociais. Busca a compreensão de um grupo ou coletividade acerca de determinados fenômenos, a partir de comportamentos, condutas, discussões e opiniões (SANTOS, DIAS, 2015).

Essas representações sociais se caracterizam por novas formas de comunicação, elaboradas nos espaços sociais, como meios de comunicação em massa, internet, igrejas e escolas (MOSCOVICI, 2017). São características das Representações Sociais, com intuito de vincular o sujeito ao objeto, a representação de algo (objeto) e alguém (sujeito); a expressão de simbolismos e interpretação do sujeito a respeito do objeto; llustra diferentes atributos comportamentais, linguísticos e materiais, além de se tratar de um modelo de conhecimento e, por fim, possui essência auxiliadora em relação ao sujeito e seu meio (JODELET, 2002).

Neste tocante, o senso comum seria a cerne da TRS, haja vista que almeja pela compreensão de grupos sociais acerca de determinados fenômenos. Sua relevância se dá pela possiblidade de compreensão da realidade social com o objetivo de se propor ferramentas que estabeleça maior aproximação entre ciência e sociedade (MARKOVÁ, 2017; MOSCOVICI, 2017).

A coleta de dados ocorreu em uma instituição pública de ensino superior em saúde do centro-oeste paulista, pioneira no Brasil na implantação de métodos de aprendizagem ativa, com 40 professores que atuam nos cenários de Tutoria, Unidade de Prática Profissional, Núcleo de Desenvolvimento Docente e do Internato Médico (HIGA et al., 2016). Para a constituição da amostra intencional da pesquisa, utilizou-se a técnica metodológica SnowBall, costumeiramente utilizada para se estudar populações difíceis de serem acessadas ou estudadas, ou que não há precisão sobre sua quantidade.

$\mathrm{Na}$ apresentação dos resultados, os professores participantes foram codificados da seguinte forma: "T", em referência a "Tutor", "UPP", em referência a "Unidade Prática Profissional". "EP", em referência a Educação Permanente e "I", em referência a Internato Médico, seguidos de sequência numérica crescente "T1", "EP 2" e "UPP 3" e "I4".

As entrevistas individuais ocorreram no local de trabalho dos participantes, agendadas previamente, respeitando a disponibilidade do participante, a partir de questões direcionadoras, no período de fevereiro de 2020 e agosto de 2020. Considerando também a pandemia da COVID 19, as entrevistas foram realizadas por via online, ligações gravadas e chats. A partir de um modelo semiestruturado, foi possível obter a caracterização dos participantes em relação a idade, sexo, estado civil e tempo de atuação docente em métodos de aprendizagem ativa, além de suas compreensões acerca da continuidade do processo de aprendizagem na prática docente, a partir da questão norteadora: "Fale o que você pensa sobre a continuidade do processo de aprendizagem da sua atividade docente". 
Os dados foram analisados a partir da técnica de Análise de Conteúdo na modalidade temática. Trata-se de um caminho que analisa o conteúdo da mensagem dos participantes para maior compreensão da leitura. Suas principais funções consistem na observação, exploração das diretrizes e gerenciamento das comprovações. Esta técnica organiza-se em três etapas: Pré-análise, etapa na qual se obtém uma visão ampla do tema, considerando os princípios da exaustividade, representatividade, homogeneidade e pertinência; Exploração do material, etapa onde ocorre a categorização, respeitando os princípios de exclusão mútua, produtividade, objetividade e fidedignidade; e Tratamento dos resultados, inferência e interpretação, que consiste na análise dos produtos oferecidos, considerando a pergunta, os referenciais teóricos utilizados e os objetivos da pesquisa (BARDIN, 2016).

Esta pesquisa foi aprovada pelo Comitê de Ética em Pesquisa (CEP) que envolve seres humanos, sob o CAAE 18630719.4.0000.5413. Como preconizado pelas Resolução 466 (2013) e Resolução 510 (2016), os participantes assinaram o Termo de Consentimento Livre e Esclarecido (TCLE) antes da coleta de dados.

\section{Resultados}

Dos 40 docentes que participaram da entrevista, 22\% atuam no cenário de tutoria, 30\% no cenário de UPP, $15 \%$ no cenário de Educação Permanente e 33\% no Internato Médico. $75 \%$ eram do sexo feminino e $25 \%$ eram do sexo masculino. A faixa etária predominante foi a de 38 a 48 anos (42\%). No contexto do tempo de atuação com métodos de aprendizagem ativa, a maioria atua a mais de 20 anos (55\%).

A análise do discurso dos docentes, a partir da questão norteadora "Fale o que você pensa sobre a continuidade do processo de aprendizagem da sua atividade docente", apontou para a categoria e subcategoria descritas no Quadro 1.

Tabela 1. Categoria e Subcategoria analítica.

\begin{tabular}{cc}
\hline Categoria analítica & Subcategoria \\
\hline $\begin{array}{c}\text { Compreensão sobre a importância da } \\
\text { continuidade do processo de aprendizagem }\end{array}$ & Mecanismo de atualização \\
\hline
\end{tabular}

\subsection{Mecanismo de atualização}

Aos serem questionados sobre a importância da continuidade do processo de aprendizagem no campo docente, os entrevistados assim responderam:

"Eu acho muito oportuno né, eu acho que toda vez que a gente, qualquer profissão que a gente exerça, é importante a gente mantar a atualização conhecer o que que as pessoas estão fazendo, como estão fazendo" (T4).

"Eu acredito que as nossas reuniões de EP contribuem bastante, são importantes essas reuniões para firmar né, firmar o que cada um tá fazendo, o que cada um pensa a respeito. É muito importante, essencial mesmo para nosso desempenho docente. Uma forma de sempre estarmos nos atualizando" (T7). 
"Vejo a continuidade da aprendizagem como fundamental para a prática docente, além de nos proporcionar condições de nos atualizarmos constantemente. Na nossa prática, na vivência do dia a dia, esses aspectos são muito importantes, os de atualização, por que as coisas acontecem todos os dias, e temos que buscar maneiras, formas mesmo de se atualizar" (E6).

"Ela é extremamente importante pra que a gente possa se atualizar, refletir e aprimorar o nosso processo de trabalho. O currículo ele é dinâmico, não dá pra você ficar parado naquilo que você viu aí na década passada, sem discutir aí quais são as inovações, quais são as possibilidades frente as dificuldades, e as barreiras que a gente encontra no nosso processo de trabalho" (U4).

"A continuidade do o processo de aprendizagem é fundamental $p$ o desenvolvimento e desempenho profissional e pessoal. Penso que é indispensável, ainda mais trabalhando em instituições com estratégias de métodos ativos. descobrir novas estratégias e relacionar a teoria com a prática só se dá pela continuidade de minha aprendizagem. É uma forma de estar por dentro daquilo que ocorre, das novas descobertas, se atualizar mesmo" (14).

A partir da análise dos dados obtidos através das entrevistas, foi possível perceber a compreensão dos entrevistados em relação à importância da continuidade do processo de aprendizagem. Os docentes a classificaram como fundamental, essencial e oportuna, com potencial para proporcionar subsídios capazes de mantê-los sempre atualizados diante das inovações contemporâneas. Além disso, enfatizou-se o fato de a continuidade ocorrer de diversas formas, como livros didáticos e recursos tecnológicos.

De fato, a continuidade no processo de aprendizagem é indispensável na construção de um perfil docente capaz de solucionar as demandas acadêmicas e sociais, considerando os diversos contextos. Atualmente, há disponível uma ampla diversidade de estratégias para que o docente exerça o aprendizado contínuo. (MEDEIROS, HIGA, MARIN, LAZARINI, LEMES, 2020).

Porém, é relevante a contextualização dos saberes e práticas docente, por se tratar de uma via de mão dupla, onde a percepção acerca da continuidade do processo de aprendizagem consiste, de forma geral, a partir do relato dos entrevistados, em mecanismos de atualização.

Como já mencionado, a sociedade está em constante desenvolvimento e evolução, principalmente através das tecnologias digitais. Com esses eventos, modificam-se também suas necessidades. Nesse contexto, os espaços escolares precisam responder á essas necessidades, sendo a figura do docente imprescindível nesse processo. Para isso, é importante que o docente esteja em constante atualização. Em sua vivência da prática, o docente é levado a tomar decisões a partir de eventos cotidianos, sendo necessário o mesmo lançar mão de uma série de procedimentos específicos, levando-o a compreender as novidades que permeiam situações em específico. Neste tocante, torna-se relevante a reflexão acerca do perfil discente contemporâneo, onde desde a infância, a partir de estratégias de aprendizagem ativa, se constrói um perfil crítico, reflexivo e questionador (MARTINS, 2020, p.103). Diante disso, considerando a ideia de os espaços escolares não se constituírem a partir de alunos passivos, que aceitam as informações como verdades absolutas, evidencia-se a relevância da continuidade do processo de aprendizagem a partir da vertente atualizadora.

Nesse contexto, é fundamental a vertente teórico-prática para o aperfeiçoamento de sua formação a partir das necessidades surgidas em sua atividade profissional, através de um processo de aprendizagem contínua, sendo os mecanismos de atualização fundamentais para se obter êxito nesse processo (MESQUITA, MENESES, RAMOS, 2016). 
No campo das inovações, como citado, as tecnologias digitais de informação e comunicação (TDIC) figuram como importante ferramenta de atualização docente. Esses recursos tecnológicos possuem grande influência nos espaços sociais e culturais da sociedade, considerando a ideia de que as informações são transmitidas de forma rápida e com dinamismo, proporcionando movimentos de interações que refletem de forma direta nas maneiras de pensar e agir da sociedade (MENDONÇA, 2020).

Nessa perspectiva educacional, faz-se necessária a atualização para manejo dessas tecnologias, considerando o atual perfil discente e seu elo com o mundo virtual. A não valorização das tecnologias nos espaços escolares contribui para o estabelecimento de uma fronteira ainda maior entre docente e discente no que tange ao processo educacional. Vale ressaltar que as TDIC, além de mostrarem-se como importante opção de capacitação e atualização docente, seu uso em sala de aula possui potencial para tornar o ensino ainda mais prazeroso (NUNES, OLIVEIRA, 2016).

Para que isso ocorra, é necessário se pensar os aspectos estruturais das instituições de ensino, além das condições ofertadas aos docentes para se capacitarem, sendo necessários reajustes nos sistemas de distribuição e comunicação das tecnologias. Reitera-se a necessidade de se pensar em flexibilizações nos contextos administrativos para que se torne viável a inserção e apropriação dessas tecnologias (JÚNIOR, 2018).

Nesse contexto de atualização docente por intermédio das tecnologias, consideram-se três aspectos que evidenciam as particularidades na realidade destes profissionais: a deficiência e/ou insuficiência nos cursos de formação docente, as reformas educativas dos anos 1990, com a Lei de Diretrizes e Bases (LDB) ㄲo 9.394 de 1996, que trouxeram novas exigências no contexto de formação de professores e as consequências provenientes do avanço tecnológico, principalmente em relação às influências exercidas sobre o conhecimento e as novas concepções de ensino e aprendizagem, onde a ótica de formação continuada transcenda a simples de ideia de se inserir computadores e dispositivos tecnológicos nos espaços escolares, enfatizando a iminente necessidade de programas de capacitação dos docentes para o manejo desses recursos (NASCIMENTO, 2020).

Ainda no contexto da atualização como ferramenta para o desenvolvimento continuado, o que se observa é um processo de continuidade que se limita a dois eixos: o primeiro se relaciona a formação continuada, onde ocorre a proximidade do profissional com a atualização, enquanto o segundo, em menor proporção, é o trabalho continuado, que evidencia a atividade docente como um processo de ensinar apenas o que é necessário, visando a inserção deste futuro profissional no mercado de trabalho, caracterizando, assim, um ensino pautado em tendências pedagógicas não liberais. Diante disso, vale a ressalta da necessidade de uma qualificação docente que atenda as exigências do trabalho e da educação, pois essa qualificação impacta de forma direta a aprendizagem dos estudantes. Nesse sentido, a atualização se faz necessária (AZEVEDO, JÚNIOR, DARÓZ, 2014).

De forma geral, a ideia de atualização docente, como componente da continuidade do processo de aprendizagem, no contexto das metodologias de aprendizagem ativa, ocorre a partir dos eixos centrais dessa estratégia de aprendizagem, baseada na integração entre teoria e prática e ensino e serviço, com o intuito principal de se desenvolver capacidade de reflexão a partir de problemas reais, além do estabelecimento de ações originais e criativas que atendam aos problemas da sociedade contemporânea (MARIN et al., 2010).

Por fim, a partir dos achados na literatura, foi possível observar uma íntima relação entre continuidade do processo de aprendizagem e atualização docente. Porém, chama a atenção o predomínio de uma aprendizagem técnica nos espaços escolares, centrada em metas, incompatível com os desafios atuais, o que, por ora, ressignifica uma revisão das políticas públicas institucionais, para que seja possível fazer valer o verdadeiro sentido da continuidade do processo de aprendizagem com foco na atualização. 


\section{Conclusão}

Considerando que o objetivo central do presente estudo foi compreender as representações sociais dos docentes sobre a continuidade do processo de aprendizagem, a partir da pesquisa qualitativa e seus pressupostos, evidenciou-se que na compreensão dos entrevistados, tal fato está diretamente relacionado com mecanismos de atualização, que pode ocorrer através de livros didáticos e recursos tecnológicos. Essa necessidade de continuidade, que oportuniza a atualização, desponta como indispensável, ao se propor uma reflexão acerca do atual perfil docente. A literatura apontou para o uso das TDIC, como potencial para o docente estar em constante atualização. porém, chamou a atenção para a necessidade de se rever políticas institucionais, qualificar a estrutura e disponibilidade de equipamentos e dispositivos, além do fortalecimento de programas de educação continuada. Outro fator que emergiu nos relatos dos entrevistados e, posteriormente, ampliado a partir de buscas na literatura, foi a importância da integração teoria e prática e ensino e serviço, através de movimentos de problematização da realidade, para que ocorra um processo reflexivo e, consequentemente, proporcione ao docente formas de estar atualizado. Neste tocante, vale a premissa de que, além da ótica ampliada acerca das condições ofertadas e espaços disponíveis para formação continuada docente, são necessárias aproximações acerca da visão de mundo dos gestores e mudança de paradigmas, uma vez que estas vertentes problematizadoras se aproximam de estratégias ativas de aprendizagem, o que requer organização e rigor metodológico.

A Teoria das Representações Sociais qualificou a análise dos achados, pois evidenciou o pensamento comum dos docentes que vivenciam os desafios cotidianos $\mathrm{e}$ as necessidades de uma melhor qualificação.

Assim, os resultados reiteram a necessidade de as instituições proporcionarem condições para que ocorra a continuidade da aprendizagem docente de forma pertinente, para que dessa forma, seja oportunizado aos docentes espaços para atualização, atendendo assim as necessidades contemporâneas, que já não são mais as mesmas do século passado. Além disso, evidenciou-se a importância da pesquisa qualitativa, mostrando-se como importante ferramenta capaz de proporcionar subsídios para que ocorra progresso no campo de formação docente.

\section{Referências}

Araújo, U.F., Arantes, V.A., Danza, H.C., Pinheiro, V.P.G., \& Garbin, M. (2016). Principles and methods to guide education for purpose: a Brazilian experience. Journal of Education for Teaching, 42(5), 1-9.

Azevedo, N.P.G., Junior, F.M.B., \& Daróz, E.P. (2014). O professor e as novas tecnologias na perspectiva da análise do discurso: (DES) encontros em sala de aula. Linguagem em DIScurso, 14(1), 15-27.

Bardin, L. (2016). Análise de conteúdo. 1ed. São Paulo: Edições 70.

Delors, J. (2003). Educação: um tesouro a descobrir. 2ed. São Paulo: Cortez, Brasília, DF: MEC/UNESCO.

Falsarella, A.M. (2004). Formação continuada e pratica em sala de aula: os efeitos da formação continuada na formação do professor. Campinas (SP): Autores Associados.

Freitas, S.I., \& Pacífico, J.M. (2020). Formação continuada: um estudo colaborativo com docentes do ensino médio de Rondônia. Interações, 21(1), 141-153.

Godinho, P.A. et al. (2017). A aprendizagem baseada em problemas (ABP) como metodologia de ensino na disciplina de embriologia na visão do aluno. Acta Scientiarum, 39(3), 327-332. 
Higa, E.F.R., Moreira, H., Pinheiro, O., Tonhom, S., Carvalho, M. H., \& Bracciali L. (2018). Caminhos da avaliação da aprendizagem ativa: visão do estudante de medicina. Revista Lusófona de Educação, 40, 171-184.

Higa, E.F.R., Tapeiro, E.F., Carvalho, M.H.R., Aguilar-Da-Silva, R., Tonhom, S.F.R., \& Hafner, M.L.B.M. (2016). Avaliação do processo pedagógico em metodologia de aprendizagem ativa. Investigação Qualitativa em Educação. CIAIQ. 2016: 1.

Jodelet, D. (2002). Representações sociais: um domínio em expansão. Rio de Janeiro (RJ): Eduerj, 17-44.

Junior, C.P. (2018). Formação docente frente às novas tecnologias: desafios e possibilidades. Intermeio, 24(47), 189-210.

Lara, E.M.O. et al. (2019). O professor nas metodologias ativas e as nuances entre ensinar e aprender: desafios e possibilidades. Revista Interface, 23.

Macedo, K.D.S., Acosta, B.S., Silva, E.B., Souza, N.S., Beck, C.L.C., \& Silva, K.K.D. (2018). Metodologias ativas de aprendizagem: caminhos possíveis para a inovação no ensino em saúde. Escola Anna Nery Revista de Enfermagem, 22(3).

Marková, I. (2017). A fabricação da teoria de representações sociais. Cadernos de Pesquisa, 47, 358-375.

Martins, G. (2020). Metodologias ativas: Métodos e Práticas para o século XXI. 1. ed. IGM Editora.

Mendonça, A.A.S. (2020). As novas tecnologias e a COVID: o repensar da capacitação docente. CIET.

Medeiros, R.O., Higa, E.F.R., Marin, M.J.S., Lazarini, C.A., \& Lemes, M.A. (2020). Desenvolvimento continuado de docentes na graduação em saúde. Política, Planejamento e Gestão em Saúde, v.6. Ponta Grossa (PR): Atena Editora.

Mesquita, S.K.C., Meneses, R.M.V., \& Ramos, D.K.R. (2016). Metodologias ativas de ensino/aprendizagem: dificuldades de docentes de um curso de enfermagem. Trabalho, Educação e Saúde, 4(2), 473-486.

Minayo, M.C.S. (2013). O desafio do conhecimento: Pesquisa Qualitativa em saúde. São Paulo (SP): Hucitec.

Moscovici, S. (2017). Representações sociais: investigações em psicologia social. 11. ed Petrópolis, RJ: Vozes.

Nascimento, M.C. (2020). Formação continuada docente para a utilização das TIC no processo de ensino e aprendizagem. Research, Society and Development, 9(2).

Oliveira, B.L.C.A., Lima, S.F., Rodrigues, L.S., \& Júnior, G.A.P. (2018). Team-Based Learning como forma de aprendizagem colaborativa e sala de aula invertida com centralidade nos estudantes no processo ensino-aprendizagem. Revista Brasileira de Educação Médica, 42(4), 86-95.

Pio, D.A.M. (2017). A experiência do professor médico com métodos ativos de ensinoaprendizagem: formação permanente e gestão com mediadoras [Tese]. Botucatu (SP): Universidade Estadual Paulista Júlio de Mesquita Filho, 223 p.

Pinto, M.L., Mistro, F.Z., \& Uemura, S.T. (2016). Ensino baseado em problemas como prática pedagógica aplicada a alunos ingressantes no curso de Odontologia. Revista Abeno, 16(3), 28-35.

Resolução n. 466, de 12 de dezembro de 2012. (2013). Aprova diretrizes e normas regulamentadoras de pesquisa envolvendo seres humanos. Diário Oficial da União, Brasília, DF.

Resolução n. 510, de 7 de abril de 2016. (2016). Sobre a Ética na Pesquisa na área de Ciências Humanas e Sociais. Diário Oficial da União, Brasília, DF.

Santos, G.T., \& Dias, J.M.B. (2015). Teoria das representações sociais: uma abordagem sociopsicológica. Revista eletrônica de humanidades, 8(1), 173-187. 
Vol. 7 | Investigação Qualitativa em Educação: Avanços e Desafios

Taroco, A.P.R. M., Tsuji, H., \& Higa, E.F.R. (2017). Currículo orientado por competência para a compreensão da integralidade. Revista Brasileira de Educação Médica, 41(1), 12-21.

Teófilo, T.J. S., Santos, N.L.P., \& Baduy, R.S. (2017). Apostas de mudança na educação médica. Interface, 21(60), 177-88.

Tsuji, H., \& Aguilar-da-Silva, A. (2010). Aprender e ensinar na escola vestida de branco: Do modelo biomédico ao humanístico. São Paulo (SP): Phorte. 